\title{
No positive bone healing after using platelet rich plasma in a skeletal defect. An observational prospective cohort study
}

\author{
Joost C. Peerbooms • Joost W. Colaris • \\ Anita A. Hakkert • Mark Van Appeldorn • \\ Daniël J. Bruijn • Brenda L. Den Oudsten • Taco Gosens
}

Received: 2 April 2012 / Accepted: 7 June 2012 /Published online: 26 June 2012

(C) Springer-Verlag 2012

\begin{abstract}
Purpose Platelet rich plasma (PRP) is derived from the patient's own blood. The activated blood platelets release a cocktail of growth factors, some of which are thought to initiate and stimulate repair. We compared two groups to investigate whether the use of PRP mixed with bone chips improves bone healing in patients with a skeletal defect.

Methods In total, 41 patients were observed. One group underwent a high tibial osteotomy with the addition of PRP and bone chips in the open wedge. The other group underwent the same procedure without the addition of PRP. Six patients had
\end{abstract}

J. C. Peerbooms $(\square)$

Orthopaedic Surgery, Albert Schweitzer hospital,

Dordrecht, The Netherlands

e-mail: jcpeerbooms@yahoo.com

J. W. Colaris

Orthopaedic Surgery, Erasmus Medical Centre,

Rotterdam, The Netherlands

\section{A. A. Hakkert}

Radiology, Haga Hospital,

The Hague, The Netherlands

M. Van Appeldorn

Cardiovascular Perfusion, Haga Hospital,

The Hague, The Netherlands

\section{J. Bruijn}

Orthopedicum, Orthopaedic Surgery,

The Hague, The Netherlands

\section{B. L. Den Oudsten}

Centre of Research on Psychology in Somatic Diseases

Department of Medical Psychology, Tilburg University,

Tilburg, The Netherlands

T. Gosens

Orthopaedic Surgery, St. Elisabeth Hospital,

Tilburg, The Netherlands to be excluded because of insufficient data or they were lost to follow-up. Bone healing was studied using computed tomography scanning. The blood was sequestered and PRP was produced using a blood cell separator with a PRP software program (Electa, Sorin Group, Mirandola, Italy).

Results Analysis focused on the remaining 35 patients. At baseline, there were no differences between the two groups for age, sex and side of operation. At one week postoperatively, the bone density under $(p=0.02)$ and above the wedge was significantly lower in the PRP group than the control group $(p=0.24)$. At six weeks postoperatively, no significant differences between the treatment groups were found. At 12 weeks, the PRP group had significantly lower bone density under the wedge compared to the control group $(\mathrm{p}=0.01)$.

Conclusions We found that patients with a skeletal defect did not benefit from the application of PRP mixed with an allograft regarding bone healing.

\section{Introduction}

It can be hypothesized that mixing platelet rich plasma (PRP) with sequestered bone graft materials, might create a bioengineered graft. The result is a bone graft enriched with a high concentration of platelets releasing growth factors. Because of the viscous nature of PRP, the bone chips will stick together, thus avoiding migration of bone particles. This may be a promising technique that could support and promote bone growth and accelerate fracture healing, particularly in patients who are at risk of the development of non-unions [1]. PRP is promoted as an ideal autologous prepared biological blood derived product, which can be exogenously applied to a diversity of tissues where it releases high concentrations of platelet growth factors that enhance bone healing [2]. 
A number of studies have focused on how PRP affects osteoblasts, osteoclasts, and mesenchymal-osteoprogenitor stem cells. It is hypothesized that platelets can act as an exogogenous source of growth factors that could potentially stimulate bone formation [3].

Because of the positive effects of the use of PRP described in the literature, we used PRP in a number of cases where patients underwent a high tibial osteotomy. We compared the results with the results of the patients who where not treated with PRP. This led to an observational prospective cohort study, which examined the hypothesis that the application of a bone allograft mixed with PRP could improve bone healing in patients with a skeletal defect in the tibia.

\section{Patients and methods}

This observational prospective cohort study contains 41 patients scheduled for a medial high tibial osteotomy between October 2005 and February 2009. All procedures took place in a training hospital (HAGA Hospital, The Hague, The Netherlands) using the same surgical procedure performed by an orthopaedic consultant or a supervised senior orthopaedic resident. Criteria for participation were osteoarthritis of the medial compartment of the knee. All affected knees were screened with whole leg standing radiographs to demonstrate radiologic medial osteoarthritis. Exclusion criteria were a platelet count lower than $150 \times 109 / 1$, hemoglobin level lower than $6.5 \mathrm{mmol} / \mathrm{l}$, BMI $>33$, varus angle $>15$ degrees.

The primary endpoint was bone density above and below the opening wedge, which is used as a measurement of bone healing. High bone density reflects good bone healing [4, 5]. The osteotomy areas on the CT scans were compared with the same areas on the CT scans performed directly postoperative (the baseline CT scan). A blinded observer estimated bone healing, based on the density evaluation on the $\mathrm{CT}$ scan. Bone-graft radiographic junctions, that are the lines at the proximal and distal level where the graft had a different density compared with the host bone, were investigated.

These areas were measured and summarized for all samples in each group at each follow-up. The bone density at the proximal and distal side of the wedge was measured in Hounsfield values in a $50 \mathrm{~mm}$.sq Region of Interest (ROI).

\section{Establishment of the groups}

All the patients in our study were scheduled to have an open high tibial osteotomy with an allograft. All patients received a date for their operation in a chronological order. They were seen in the orthopaedic outpatient clinic at approximately three months prior to the operation date. The decision to operate was made in the orthopaedic outpatients clinic, without knowing if the transfusionist, who would prepare the PRP, would be available during the operation. If the transfusionist was available the patients received PRP, if he was not available they did not receive PRP. This led to two groups, which were created depending on the availability of the transfusionist, a PRP group and a control group.

\section{Surgical procedure}

A medial approach was performed, shifting the pes anserinus dorsally. A Kirschner wire was inserted just proximal of the tibial tuberosity in lateral cranial direction in order to obtain an equal osteotomy angle. Along this wire the osteotomy was performed. The lateral cortex was left intact. The gap was opened and allograft bone chips were inserted. Internal fixation was performed using the Tomofix plate (SynthesGmbh, Solothurn, Switzerland). The patient's own platelets were collected using a platelet separation system into a highly concentrated formula, PRP-fraction. By addition of calcified thrombin the platelets were activated. PRP was mixed with the allograft. Thereafter, the wound was closed in layers. After closure of the subcutaneous tissues and the skin, the layers were sprayed with the platelet poor plasma (PPP) fraction. To prevent outflow of PRP, no drain was used.

In all patients the medial incision was dressed postoperatively with compression bandages and rehabilitation was started at the day after surgery. For the first six weeks there was restricted weight bearing $(10 \mathrm{~kg})$ with two crutches. After six weeks weight bearing was allowed depending on the consolidation of the open wedge. Post-operative pain relief was achieved using a standard protocol (paracetamol $3 \mathrm{~g}$ daily and diclofenac $50 \mathrm{mg}$ three times daily with pantoprazol $40 \mathrm{mg}$ daily as an ulcer protection). All patients received thrombosis prophylaxis via a subcutaneous injection of $0.3 \mathrm{ml}$ low-molecular-weight heparin daily before operation until six weeks post-operative.

\section{Platelet rich plasma preparation}

In the pre-operative room, $450 \mathrm{ml}$ of the patients blood was drawn into a blood bag containing $63 \mathrm{~mL}$ of citrate phosphate dextrose formula A. The blood was sequestered and PRP was produced using a blood cell separator with a PRP software program (Electa, Sorin Group, Mirandola, Italy). In brief, blood was drawn into a $175 \mathrm{~mL}$ bowl of the blood cell separator and centrifuged at $1660 \mathrm{~g}$ (5600 RPM) for sequestration. After sequestration, $12 \mathrm{~mL}$ of platelet poor plasma was collected in an Activat transfer syringe (Activat, Sorin Group, Mirandola, Italy) and processed according to manufacturer instructions to prepare autologous thrombin. During 
PRP production, centrifugation was reduced to $350 \mathrm{~g}$ (2400 RPM). Approximately $30 \mathrm{~mL}$ of PRP and white blood cells were collected in a $50 \mathrm{~mL}$ syringe. The remaining blood components were collected in a blood bag and transfused during surgery.

In the operating room three $20 \mathrm{~mL}$ syringes with open top end were filled with bone chips (cancellous chips $30 \mathrm{cc} /$ 0.17-1.0 cm, NBF Dutch Bone Bank Foundation, OsteotechInc, New Jersey, U.S.A.). PRP were activated with thrombin on a 1:10 ratio, filling the $20 \mathrm{~mL}$ syringes containing bone chips making it a bone log. The surgeon applied the bone logs on the implants and tissues.

\section{Computed tomography scanning}

As a CT scanner we used a Toshiba Aquillion 16 scanner. The patient was put in a supine position on the CT couch. An Anterior-Posterior scan was made. Helical $0.5 \mathrm{~mm} \mathrm{CT}$ sections were obtained of the side which was operated on, at a pitch of 1: $1.25(120 \mathrm{kV}, 250 \mathrm{~mA}$, rotation time $0.5 \mathrm{~s}$, scan field-of-view $32 \mathrm{~cm}$, bone and standard algorithm). From the helical CT data, we reconstructed thin axial slices at $0.5 \mathrm{~mm}$ intervals to yield near-isotropic voxels (almost identical to the length of the voxel $\mathrm{x}, \mathrm{y}$, and $\mathrm{z}$ axes) for further processing. This allowed us to make two-dimensional and three-dimensional reconstructions, with a resolution similar to the source images. This formed the basis of good-quality multiplanar reconstructions (MPRs).

From October 2005 until February 2009 the obtained data were archived in the CederaMinipacs as well as on MOD (Magneto Optical Disk) Sony EDM-2600B 2.6 GB. Later on this data was imported into the Philips iSitePacs. All examinations, which took place after digitalization (September 2007), were archived directly in the iSitePacs.

For reading the scans the TerareconAquarisnet software was used. To use this, the scans were exported from the Pacs to the Terarecon server. The $0.5 \mathrm{~mm}$ series was uploaded into the Terarecon program. At first, the complete series of transverse images were viewed in craniocaudal direction in order to obtain an opinion on the entire area, which had been operated on. At the height of the fibular head an oblique line is chosen, running from the most ventral point of the osteosynthesis material to the center of the fibular head (Fig. 1). Angulated at this line the images are viewed in an oblique plane (Fig. 2).

The reading of the bone density at the junction cranial and caudal from the wedge was measured in Hounsfield values in a $50 \mathrm{~mm}^{2}$ ROI (Fig. 3). The position of the ROI was chosen in a way that beam-hardening artefacts from the osteosynthesis material would not influence the measurement of the ROI. An independent research assistant obtained measurements in scans postoperatively, which were made within one week postoperatively, six weeks postoperatively and three months.

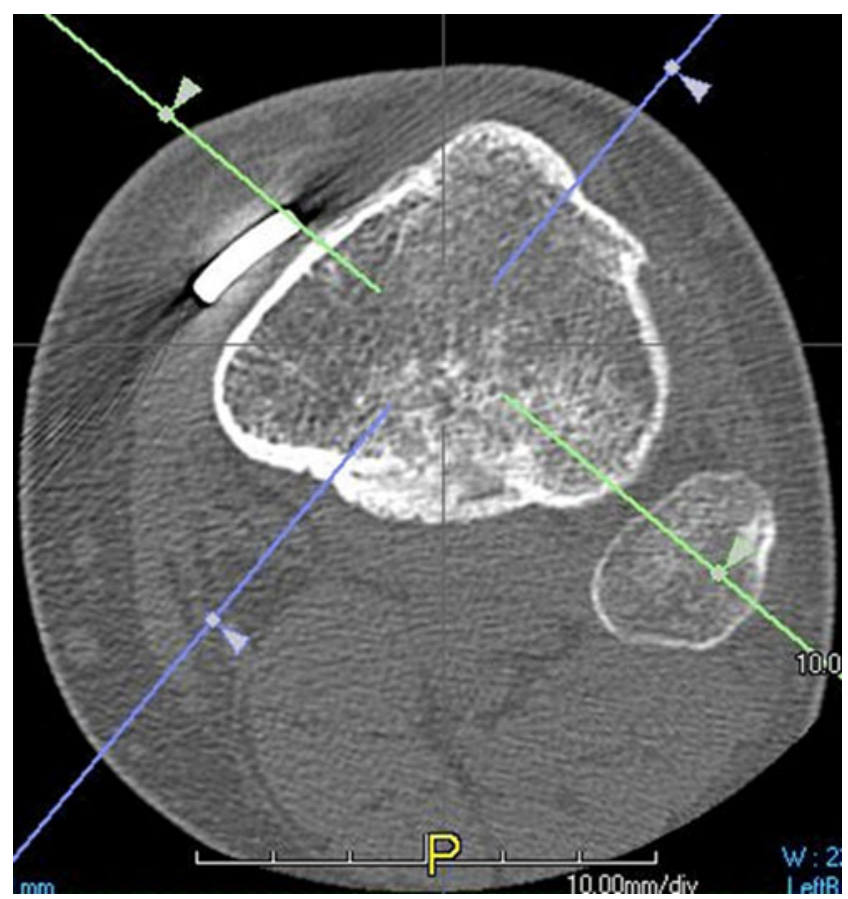

Fig. 1 In the transverse plane, at the height of the fibular head, an oblique line is, running from the most ventral point of the osteosynthesis material to the center of the fibular head (green line). A perpendicular line (purple line) is drawn in the center of the tibia

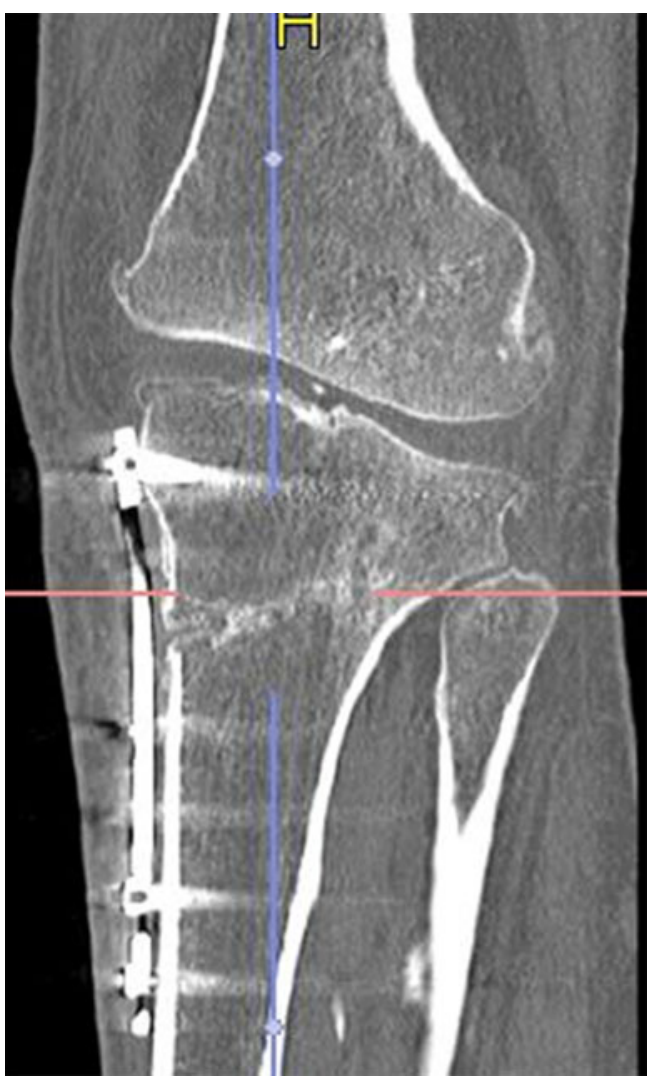

Fig. 2 Angulated at the purple line, the images are viewed in an oblique plane. The red line is perpendicular to the purple line 


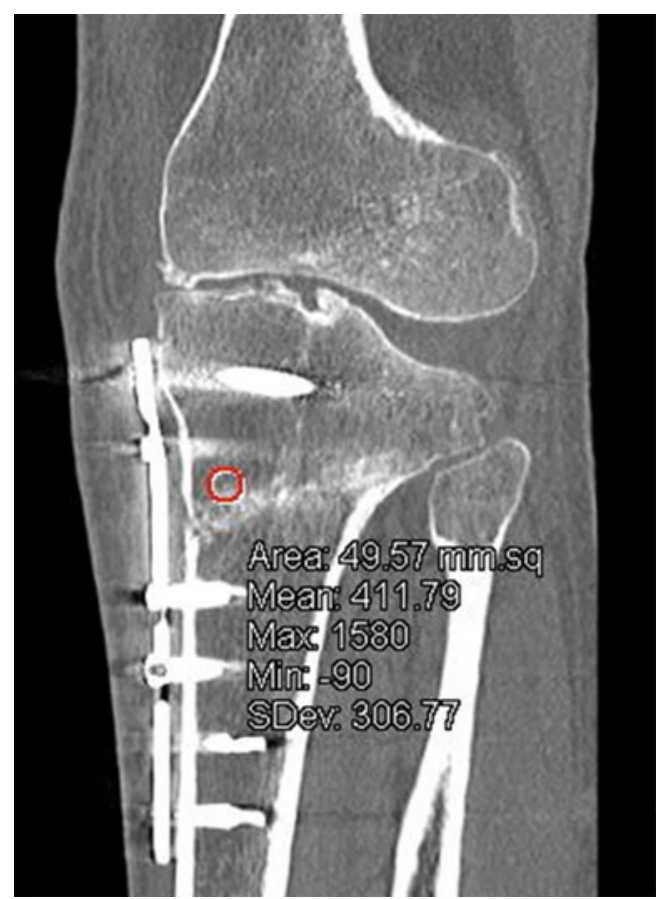

Fig. 3 The reading of the bone density at the junction above and below the wedge is measured in Hounsfield values in a $50 \mathrm{~mm}^{2}$ ROI (intersection of red and purple line)

\section{Statistics}

The main aim was to evaluate changes in bone density (above and under the wedge) in the two groups and to compare these groups at baseline, six weeks postoperative, and 12 weeks postoperative. Descriptive statistics were used to present the available sociodemographic and clinical data at baseline.

The Mann-Whitney U test and Chi-square test were used to examine patients who completed the study and those lost to follow-up regarding patient characteristics (i.e., age, sex, side of operation). In addition, Mann-Whitney U tests were used to assess statistical differences at all time points (one week, six weeks, and 12 weeks after treatment) between the PRP group and the control group. The Monte Carlo method, which involves creating a distribution similar to the sample distribution by taking several samples (i.e., 10,000 samples) from this distribution, was used to estimate the confidence intervals. Because of multiple comparisons, these tests were corrected with a Bonferonni correction for the number of tests performed $(0.05 /$ number of comparisons). Thus, rather than using $\mathrm{p}<0.05$, the critical level of significance was 0.02 .

A global data analysis to determine whether patients improve or deteriorate was performed with Friedman's ANOVA, which were used to compare the bone density scores above and under the wedge at all time points. When significant results were found, post-hoc tests were used to determine which time points differ from another. Since the Friedman's ANOVA can only be used in a 'one-within' design, the data set was split into PRP group and control group in order to examine the course of bone density across time in these groups.

The level of statistical significance was set at $\mathrm{p}<0.05$ (two-tailed). All statistical analyses were performed using the Statistical Package for the Social Sciences (SPSS Chicago, IL, USA, version 17.0).

\section{Results}

From October 2005 until February 2009, 41 patients with high medial tibial osteotomies underwent surgery. Twenty patients were treated with PRP and 21 without PRP. The cohort consists of 16 males and 25 females with an average age of 51.0 years $(\mathrm{SD}=7.3)$. The left-right ratio was 21:20. Nineteen patients received PRP (PRP group), while the other patients did not receive PRP (control group). From the PRP group, one patient was excluded due to insufficient registration of data. From the control group, five patients were excluded for similar reasons. Analysis between patients who completed the study and those who were lost to follow-up did not show any differences in patient characteristics (i.e., age, sex, side of operation; $p>0.46$ ). At one week postoperatively, there were no differences found between the PRP group and the control group (i.e., age, sex, side of operation; ps $>0.46$ (Table 1$)$ ).

\section{Bone density under the wedge}

Across time, the level of bone density did not change when the total group (PRP + control group) was analyzed $(p=$ 0.14 ) or when both treatment groups were analyzed separately $[\mathrm{p}=0.17$ (control group) and $\mathrm{p}=0.61$ (PRP group)].

Figure 4 shows the bone density under the wedge for both PRP and the control group at different time points. At one

Table 1 Patient characteristics

\begin{tabular}{llll}
\hline Characteristics & PRP group N=19 & Control group N=21 & P-value (95 \% CI) \\
\hline Age (years) & $52.1(\mathrm{SD}=6.4)$ & $49.9(\mathrm{SD}=8.0)$ & $0.46(0.46-0.48)$ \\
Sex (male) & $7(36.8 \%)$ & $8(43.8 \%)$ & 0.74 \\
Side of operation (left) & $11(57.9 \%)$ & $7(43.8 \%)$ & 0.51 \\
\hline
\end{tabular}


Fig. 4 Mean scores of bone density under the wedge for the PRP group and the control group at one week, six weeks, and 12 weeks after treatment

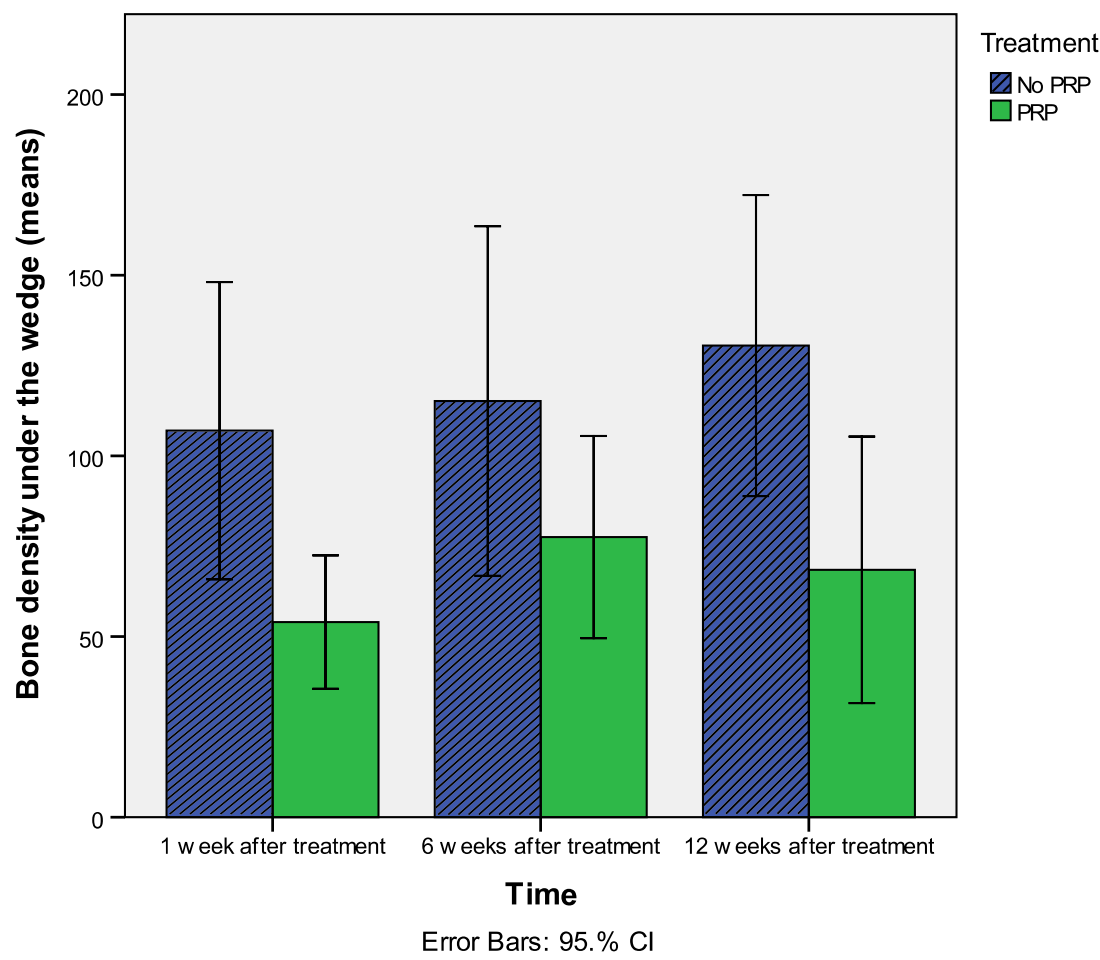

week postoperatively, the bone density under the wedge was significantly lower in the PRP group 50.8 (SD 37.8) than the control group 119.0 (SD 94.6; $\mathrm{p}=0.02$ ). At 6 weeks postoperatively, no significant differences between the groups were found ( $\mathrm{p}=0.13$ ). At 12 weeks, the PRP group 68.5 (SD 74.1) had significantly lower scores than the control group 129.1 (SD 85.7; $\mathrm{p}=0.01$ ).

Bone density above the wedge

Across time, the level of bone density improved when the total group (PRP + control group) was analyzed ( $\mathrm{p}=$ 0.03 ). At one week postoperatively, the mean density was 102.5 (SD 76.0) and after three months the density was 140.4 (SD 96.7). However, when the analyses across time were replicated, in which both treatment groups were analyzed separately, improvements were present, although no significant change was found. The bone density above the wedge improved from 132.8 (SD 86.8) to 167.7 (SD 111.6; $\mathrm{p}=0.19$ ), while the PRP group improved from 77.1 (SD 55.9) to 117.5 (SD 77.8; $\mathrm{p}=0.13$ ).

Figure 5 shows the bone density above the wedge for both PRP and the control groups at different time points. No significant results were found regarding the bone density above the wedge, except for the bone density assessed at one week postoperatively $(p=0.02)$. The control group 132.8 (SD 86.8) had higher scores than the PRP group 77.1 (SD 55.9).

\section{Discussion}

The purpose of our cohort study was to test the application of platelet rich plasma (PRP) in combination with an allograft in patients with a skeletal defect. Peerbooms et al. [6, 7] showed that PRP is safe and easy to use in their studies, but it does not always have a beneficial effect. This trial showed no statistical significant beneficial effect of the use of the PRP at 12 weeks after treatment when comparing the bone density with the bone density of the control group.

In the literature a diversity of filling material is described, which can be used to place in a skeletal defect. For example, bone-cement, hydroxyapatite, calciumtriphosphate and autologous bone are used $[8,9]$. However, the last option is not so attractive due to the amount of comorbidity after the harvest of autologous bone $[10,11]$. This comorbidity might be systematically overemphasized [12].

PRP is promoted as an ideal autologous prepared biological blood derived product, which can be exogenously applied to a diversity of tissues where it releases high concentrations of platelet growth factors that enhance bone healing. In addition, it possesses antimicrobial properties that may contribute to the prevention of infections [2].

At bone fracture sites, platelets release PDGF, TGF- $\beta$, and EGF, providing an ideal system for delivery of growth factors to the injury site. The richest source of TGF- $\beta$ is found in platelets, bone, and cartilage. Two isoforms, TGF$\beta 1$ and TGF- $\beta 2$, are present in the platelets. TGF- $\beta 1$ has the greatest potential for bone repair since both chondrocytes 
Fig. 5 Mean scores of bone density above the wedge for the PRP group and the control group at one week, six weeks, and 12 weeks after treatment

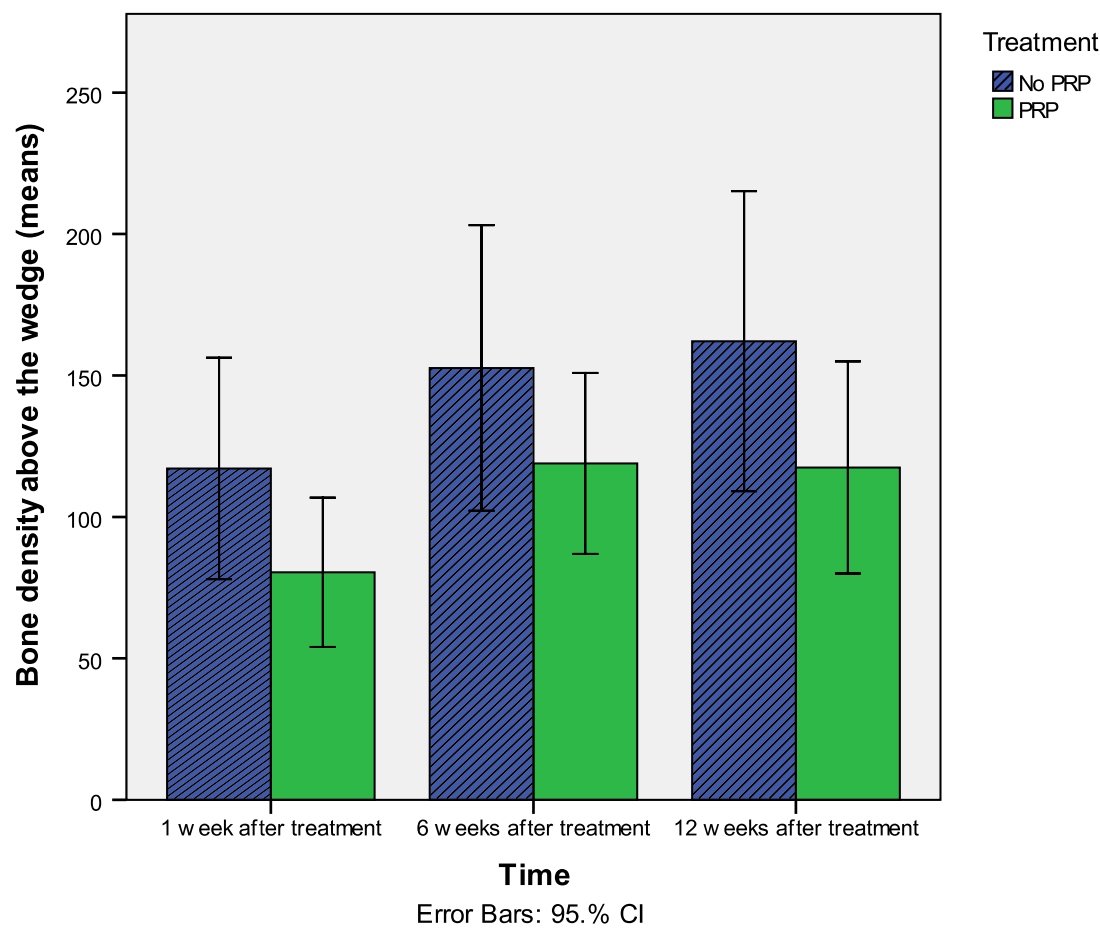

and osteoblasts are enriched with receptors for TGF- $\beta 1$. In fact, TGF- $\beta$ may contribute to bone healing at all stages. It has been demonstrated that with a combination of platelets growth factors TGF- $\beta$, FGF, and EGF, an optimum is created for the stimulation of differentiation and proliferation of osteoblasts to osteogenic cells [1].

Autologous bone graft combined with PRP has been shown to accelerate bone healing and remodeling following long bone fractures in animal models [5, 12]. It also has shown to improve osteochondral healing in a rabbit model [13]. Other animal studies found that the use of PRP in combination with bone graft material was not superior to autologous bone graft alone [14]. Conflicting results regarding the effectiveness of PRP may be partly due to the wide variation in the way PRP is produced [15]. The amount of platelets required for a positive effect from the platelet rich plasma seems to span a relatively low range of concentrations, from approximately 500,000 to $1,800,000$ platelets (median, 1,000,000 platelets) per microliter of platelet rich plasma. Below this range, the effect of platelet rich plasma is suboptimal. Also, beyond this range, particularly for concentrations of $>2,000,000$ platelets per microliter of PRP, an inhibitory effect has been observed on bone regeneration and on osteoblast activity [5]. Still, there is no standardized protocol for "the best" PRP production. There is also a lack of knowledge about the most perfect period for the activity of each agent that this PRP product contains. Several PRP systems are now available that allow an efficient preparation for outpatient use. Differences such as volume of autologous blood, spin rate, activating agent, leukocyte concentration, final PRP volume, and final platelet concentration and growth factor, characterize the systems which are available. Haematological variation between patients (e.g., the number of leukocytes, platelets) may also affect the final PRP preparation. Regarding the optimal amount of platelets and growth factors necessary for the healing of musculoskeletal injuries and support of the discussion remains exist. PRP has a clinical effect only if at least four times the normal concentration of platelets [16]. However, the efficacy of PRP is shown in less concentrated amounts [17]. PRP at $10 \%$ interfered with the complete differentiation process of human osteoclast precursors. At higher concentration it impaired osteoclast formation also at an early stage of differentiation [18]. Recent studies emphasize the effect of PRP on IL-1. This cytokine has been described to induce the production of destructive proteases together with the inhibition of extracellular matrix formation. Platelet rich plasma releasate counteracts effects of an inflammatory environment on genes regulating matrix degradation and formation in human chondrocytes [19].

Regarding the radiographic analysis, we used a score that depended on the density evaluation as an indirect index of bone healing. We choose to use computed tomography as a diagnostic tool to score bone density after high medial tibial osteotomy. Bhattacharyya et al. [20] described that a CTscan has a good diagnostic accuracy (89.9\%) in the evaluation of tibial fracture healing. Also, Markel et al. [21] described the benefits of the use of a CT-scan for evaluation of the local properties of bone in a dog model. Dallari et al. [5] and Guerrero et al. [4] used bone density measurements in their studies to score the healing of the osteotomy site. It seems to be more logical to measure a ROI in the middle of the defect, but this makes the measurements less accurate. 
Most of the ROIs would overlap the area above the wedge, the wedge itself and the area under the wedge. Therefore, we chose the ROIs at the junction above and below the defect, which provided us with the most accurate measurements, on which we could make conclusions of the bone healing.

Also, the presentation of the results from a very specific way of CT measurement has a lack of two-separated intraand inter-observer evaluation.

Another limitation is the amount of patients in our study. The reason why we have a small amount of patients is that we first wanted to do a cohort study to test if PRP would be of any advantage. We chose to exclude all patients who did not have a complete follow-up. We must conclude that this study was underpowered. As a consequence, two problems may be present: (i) when significant results are present, we may have generalization problems and (ii) when results are not significant this may be due to the fact that the study is underpowered. If you compare the length of follow-up with other studies we had a relatively short follow-up [5]. To be more accurate follow-up should be at least one year with more patients.

In our study, one patient had a non-union. This patient was treated without PRP. In a second operation PRP was used and bone healing occurred within three months.

In our patients, the final clinical outcome of bone chips with the addition of PRP did differ from that obtained with the use of bone chips alone. The PRP group showed an inhibitory effect regarding the bone density under the wedge. Therefore, we do not recommend using PRP in patients who are about to have an open tibial osteotomy.

Further randomized studies are needed to investigate the use of PRP in larger skeletal defects.

\section{References}

1. Everts P, Knape J, Weibrich G et al (2006) Platelet rich plasma and platelet gel. A review. J Extra Corpor Technol 38:174-187

2. Everts P, Overdevest E, Jakimowicz J et al (2007) The use of autologous platelet-leukocyte gels in enhancing the healing process in surgery, a review. Surg Endosc 21(11):2063-2068

3. Foster T, Puskas B, Mandelbaum B et al (2009) Platelet-rich plasma: from basic science to clinical applications. Am J Sports Med 37(11):2259-2272, Review

4. Guerrero T, Makara M, Katiofsky K et al (2011) Comparison of healing of the osteotomy gap after tibial tuberosity advancement with and without use of an autogenouscancellous bone graft. Vet Surg 40(1):27-33

5. Dallari D, Savarino L, Stagni C et al (2007) Enhanced tibial osteotomy healing with use of bone grafts supplemented with platelet gel or platelet gel and bone marrow stromal cells. J Bone Joint Surg [Am] 89(11):2413-2420

6. Peerbooms J, de Wolf S, Colaris J et al (2009) No positive effects of Autologous Platelet Gel after Total Knee Arthroplasty. A blind randomized controlled study. Acta Orthop 80(5):557-562

7. Peerbooms J, Sluimer J, Bruijn D, Gosens T (2010) Positive effect of autologous thrombocytes in lateral epicondylitis. A double blind randomized controlled trial: PRP versus corticosteroid injection. AJSM 38:255-262

8. Koshino T, Murase T, Saito T (2003) Medial open wedge high tibial osteotomy with use of porous hydroyapatite to treat medial compartment osteoarthritis of the knee. J Bone Joint Surg [Am] 85A(1):78-85

9. Redja B, Peelen J, de Groot K (1977) Tri-calcium phosphate as a bone substitute. J Bioeng 1(2):93-97

10. Heary R, Schlenk R, Sacchieri T et al (2002) Persistent iliac crest donor site pain: independent outcome assessment. Neurosurgery 50(3):510-516

11. Silber J, Anderson D, Daffner S et al (2003) Donor site morbidity after anterior iliac crest bone harvest for single-level anterior cervical discectomy and fusion. Spine 28(2):134-139

12. Carragee J, Comer G, Smith M (2011) Local bone graft harvesting and volumes in posterolateral lumbar fusion: a technical report. Spine J 11(6):540-544

13. Sun Y, Feng Y, Zhang $C$ et al (2010) The regenerative effect of platelet-rich plasma on healing in large osteochondral defects. Int Orthop 34(4):589-597

14. Jensen T, Rahbek O, Overgaard S, Søballe K (2004) Platelet rich plasma and fresh frozen bone allograft as enhancement of implant fixation. An experimental study in dogs. J Orthop Res 22(3):653-658

15. Anitua E, Sánchez M, Nurden A et al (2006) New insights into and novel applications for platelet-rich fibrin therapies. Trends Biotechnol 24(5):227-234

16. Marx R (2001) Platelet-rich plasma (PRP): what is PRP and what is not PRP? Implant Dent 10(4):225-233

17. Sánchez M, Anitua E, Azofra J et al (2007) Comparison of surgically repaired Achilles tendon tears using platelet-rich fibrin matrices. Am J Sports Med 35:245-251

18. Cenni E, Avnet S, Fotia C et al (2010) Platelet-rich plasma impairs osteoclast generation from human precursors of peripheral blood. J Orthop Res 28(6):792-797

19. van Buul G, Koevoet W, Kops N et al (2011) Platelet-rich plasma releasate inhibits inflammatory processes in osteoarthritic chondrocytes. Am J Sports Med 39(11):2362-2370

20. Bhattacharyya T, Bouchard K, Phadke A et al (2006) The accuracy of computed Tomographay for the diagnosis of tibial nonunion. J Bone Joint Surg [Am] 88-A(4):692-698

21. Markel M, Wikenheiser M, Morin R et al (1990) Quantification of bone healing: Comparison of QCT, SPA, MRI, and DEXA in dog osteotomies. Acta Orthop Scand 61(6):487-498 